\title{
Evolución del comportamiento de los estudiantes de Biomecánica en el campus virtual
}

$M^{a}$ Teresa Angulo-Carrere ${ }^{a}$, Yolanda Fuentes-Peñaranda ${ }^{a}$, Ana María Álvarez-Méndez ${ }^{a}$, Carmen Bravo-Llatas ${ }^{\mathbf{b}}$, Jesús Cristobal Barrios ${ }^{\mathfrak{c}}$ y María Pilar Álvarez Vázquez ${ }^{\mathrm{d}}$

aDepartamento de Enfermería, Facultad de Enfermería, Fisioterapia y Podología, Universidad Complutense de Madrid,

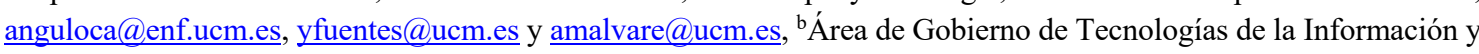
de Apoyo Técnico al Usuario, Universidad Complutense de Madrid, mcbravo@ucm.es ${ }^{c}$ Área de Software Corporativo, Universidad Complutense de Madrid, jcristobal@ucm.es y ${ }^{\mathrm{d} S e c c i o ́ n}$ departamental de Biología Celular, Facultad de Medicina, Universidad Complutense de Madrid pilar@med.ucm.es

\section{\$EWWFW}

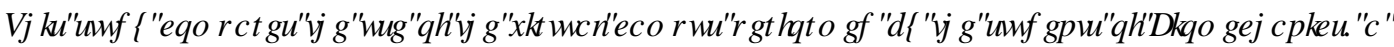

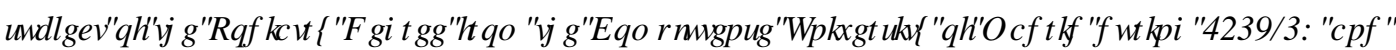

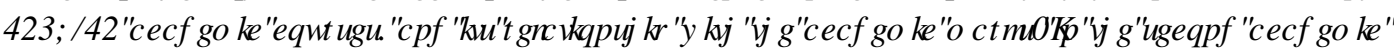
FRXUYHUWDKKQJ

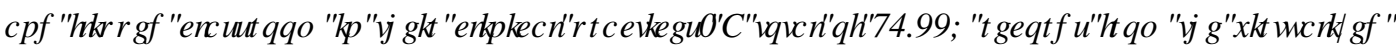

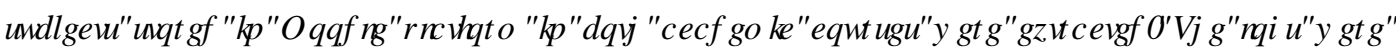

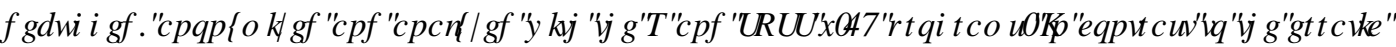

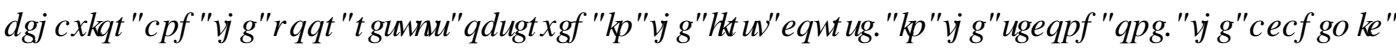

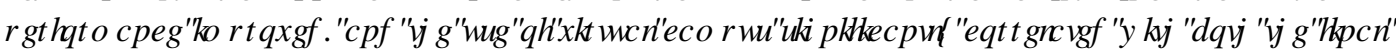

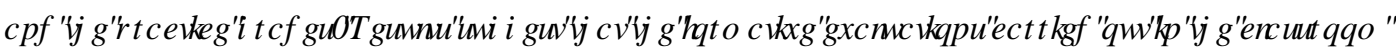

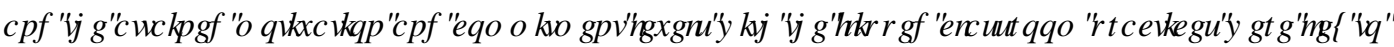

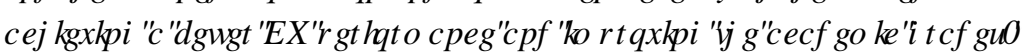

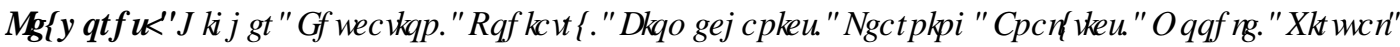

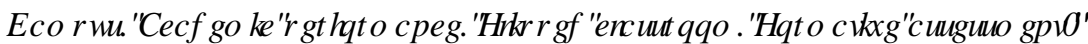

\section{HXP HQ}

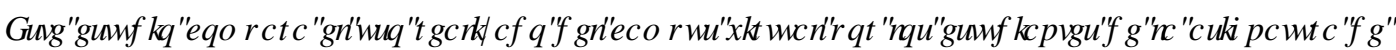

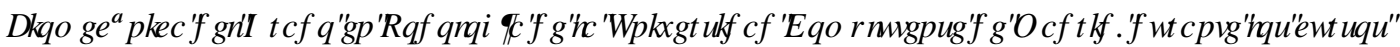

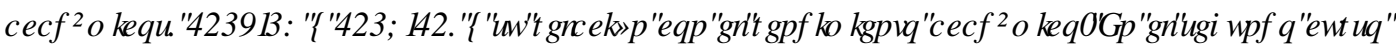

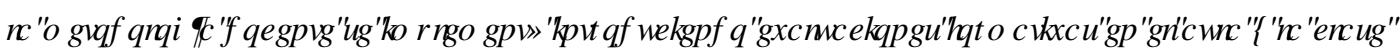

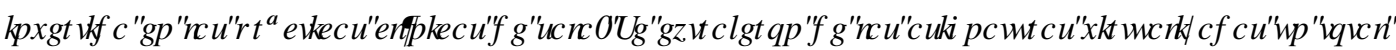

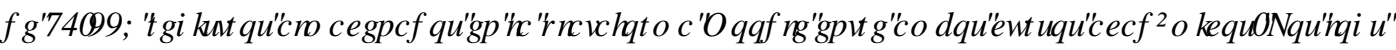

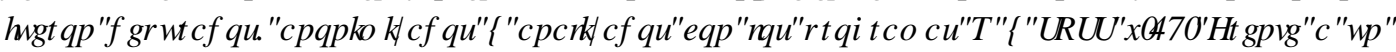

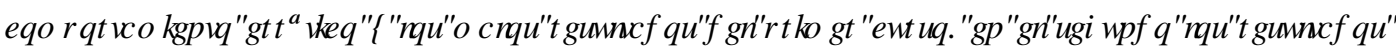

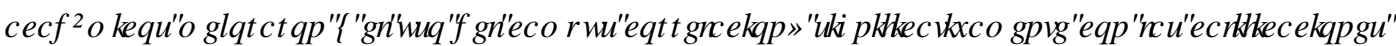

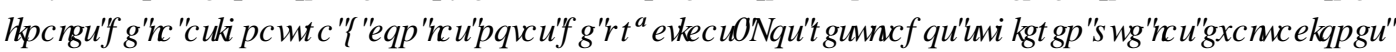

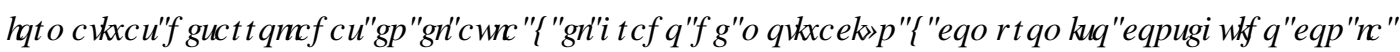

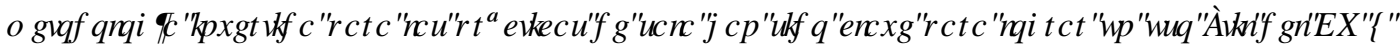
P HKUUHOUHQOP IHQRRLFDQP IFR]

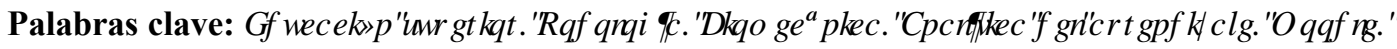

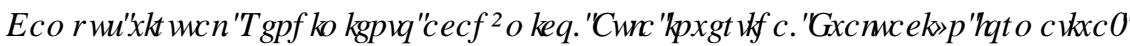




\section{Introducción}

El Espacio Europeo de Educación Superior y la puesta en marcha de los planes de estudio basados en la Declaración de Bolonia han supuesto cambios profundos en el proceso de enseñanza-aprendizaje, poniendo al estudiante en el centro del proceso y promoviendo su participación activa, de modo que deje de ser un mero receptor de información y memorizador de datos para convertirse en el actor principal responsable de su propio aprendizaje (Chao, 2014). En ese proceso de transición desde el modelo de educación tradicional, las Tecnologías de la Información y la Comunicación (TIC) han jugado un papel clave. El uso de entornos virtuales de aprendizaje no solo facilita la comunicación y la interacción entre usuarios, y la búsqueda, análisis y reelaboración de la información (Vinueza y Morocho, 2017), sino que permite un uso flexible en el espacio y en el tiempo, así como la monitorización de cada usuario (Salinas, 2004). Hay estudios, como el de Popescu et al. (2019), que muestran las preferencias de los estudiantes universitarios por actividades de aprendizaje más visuales, más interactivas y de aplicación práctica, con experiencias de aprendizaje más digitales. Sin embargo, sabemos que el uso de las TIC en la docencia también presenta algunas desventajas. Pueden generar distracción y dispersión en el estudiante, originar aprendizajes incompletos y/o superficiales, propiciar la pereza e incluso causar aislamiento y ansiedad. Estos inconvenientes obligan a los docentes a diseñar los espacios virtuales con objetivos específicos integrados en un marco de acción pedagógico (Chao, 2014; Granados-Zúñiga, 2019; Quirós, 2009). Hay que tener en cuenta también las características de las últimas generaciones de estudiantes universitarios, pues representan auténticos desafíos para los docentes: sus períodos de atención son muy cortos, su conducta es multitarea y suelen presentar una falta general de pensamiento crítico (Popescu et al, 2019). El uso de aulas virtuales con sus diversos recursos audiovisuales e hipertextos podrían representar un factor de distracción que reduzca la comprensión y el aprendizaje, comparado con aquellas personas que practican lecturas lineales de textos impresos. La gran mayoría de los universitarios no tienen la capacidad de orientarse correctamente en la búsqueda y no saben discriminar para seleccionar la mejor información (García, 2017). Este contexto obliga a los docentes a adaptar las metodologías didácticas de forma creativa.

El análisis de los registros almacenados en las plataformas digitales proporciona información valiosa sobre las actividades desarrolladas (Huapaya et al., 2012). Se ha demostrado en muchos casos una relación directa entre la participación de los estudiantes en el campus virtual (CV) y el rendimiento académico (Pernías, 2011; Sael et al., 2013). No obstante, también se han descrito casos en los que los resultados son dispares o no concluyentes (Borgobello y Roselli, 2016; Vinueza y Morocho, 2017). En este sentido, en anteriores estudios obtuvimos resultados que mostraban claras diferencias entre el uso del CV y el resultado académico obtenido por los estudiantes en dos asignaturas similares en el diseño del CV y en contenidos, e impartidas de manera equivalente por el mismo docente, en dos grados distintos de la Universidad Complutense de Madrid (Angulo et al., 2020). En concreto, ese estudio reveló un mayor uso del CV, pero calificaciones peores en estudiantes de la asignatura de Biomecánica (BM) del Grado en Podología, en comparación con el uso y resultados logrados por los estudiantes de otra asignatura, Anatomía Humana III del Grado en Fisioterapia. Con la finalidad de mejorar el rendimiento académico del alumnado de la asignatura BM, se introdujeron cambios en la metodología docente.

\section{Objetivos}

El objetivo del estudio fue doble. En primer lugar, comprobar si las innovaciones introducidas en la metodología didáctica en BM en el curso 2019/20 produjeron mejoras en el rendimiento académico. En segundo lugar, analizar si dichos cambios afectaron o no al comportamiento de los estudiantes en el uso del CV. 


\section{Desarrollo de la innovación}

La BM es una materia obligatoria, con 6 ECTS, impartida en el segundo curso del Grado en Podología en la UCM. Su docencia tiene lugar en el primer semestre del curso, iniciándose a primeros de septiembre y acabando en el mes de febrero. Existen dos convocatorias de examen final, la primera de ellas tiene lugar en febrero y la segunda, en julio. La parte teórica se organiza en 5 bloques didácticos, cuatro de los cuales se evalúan mediante cuestionarios en el $\mathrm{CV}$ y con un examen final teórico. La parte práctica se desarrolla en la sala de prácticas y se evalúa con la participación del estudiante y un examen final práctico.

Los espacios digitales de BM se virtualizaron en la plataforma Moodle, manteniendo el mismo diseño, recursos y herramientas en ambos cursos académicos 2017/18 y 2019/20. En ambos se incluyeron herramientas de comunicación (foro de avisos, correo y mensajería) y para cada unidad temática se creó un foro general para plantear y resolver dudas sobre sus contenidos. Además de archivos informativos (guía docente, horarios de tutorías, normativa, etc.) se pusieron a disposición del alumnado distintos recursos (presentaciones en Power Point, archivos en PDF, enlaces a URL formativas y a vídeos de YouTube) para facilitar el aprendizaje de los contenidos teóricos y prácticos. En el curso 2019/20, la docencia se implementó haciendo evaluaciones formativas en la parte teórica mediante autoevaluaciones en CV (Martínez, 2012; Mallén y Domínguez, 2014) al acabar cada unidad didáctica del temario, haciendo hincapié en los contenidos y conceptos más importantes de cada parte de la materia. En la parte práctica se introdujo la metodología del aula inversa (Álvarez et al., 2018; Prieto-Martín et al., 2019) de forma que, después de la docencia teórica de cada unidad temática, el alumnado recibía en el CV, una semana antes de realizar la correspondiente clase práctica, un breve resumen de las técnicas de exploración de cada complejo articular (materiales elaborados específicamente para ello a través de un proyecto de innovación docente), indicándole que su estudio era obligatorio ya que la evaluación del aprendizaje tendría lugar en la propia sala de prácticas. Las dudas que pudieran surgir serían contestadas a través del foro general de dudas. El día de la práctica, se distribuía al alumnado en grupos y se asignaban diferentes casos clínicos relacionados con la temática desarrollada en el material que se había facilitado en el CV. Las cuestiones, discusiones y correcciones de las técnicas a utilizar en cada caso eran consensuadas intra e intergrupos bajo supervisión de la profesora responsable.

Para realizar el análisis del uso de asignatura virtualizada en el curso 2019/20, se extrajeron los registros almacenados en la plataforma Moodle. Un total de 23.655 registros o $\mathbb{R} J V$ se depuraron, anonimizaron, procesaron y estandarizaron, excluyendo aquellos que no correspondieran a participantes con el rol de estudiantes. Estos $\mathbb{R} J V$ fueron analizados mediante el lenguaje de programación R y con RStudio. Este software, de uso libre y gratuito, es ampliamente utilizado en el entorno universitario, pues ofrece la posibilidad de utilizar diferentes paquetes con funcionalidades de cálculo y gráficas, lo que permite realizar análisis profundos y muy sofisticados (Ferrero y López, 2018; Ollé, 2019). Las variables estudiadas fueron el número de visitas diarias, la frecuencia de accesos a recursos y a URLs, y el número de cuestionarios completados. Los datos relativos al rendimiento académico fueron facilitados por la docente encargada de la asignatura y tratados de forma anonimizada. Según las calificaciones obtenidas, los estudiantes se segregaron en 3 categorías: notas inferiores a 5 ; notas entre 5 y 8 ; y calificaciones superiores a 8 .

Para el análisis estadístico de los datos se utilizó el programa informático IBM SPSS v.25, incluyendo correlaciones paramétricas de Pearson y no paramétricas de Spearman, árboles de decisión mediante el criterio de corte CHAID (minimización de la significatividad del estadístico de Fisher-Snedecor) para relacionar grupos de estudiantes con una actividad similar en CV con el rendimiento académico. 


\section{Resultados}

En el curso académico 2019/20 se matricularon en la asignatura BM 83 estudiantes, siendo el 67,5\% mujeres. El espacio virtualizado fue frecuentado por el 100\% del alumnado. En la Tabla 1, se recogen los estadísticos del uso del CV en los cursos académicos comparados. Puede observarse un drástico descenso en los accesos en el segundo curso que se acompaña de subidas más discretas en el uso de recursos y de URLs.

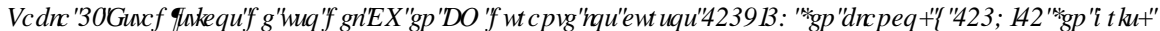

\begin{tabular}{l|cc|cc|cc}
\hline \multicolumn{3}{c}{ Visitas } & \multicolumn{3}{c}{ Recursos } & \multicolumn{2}{c}{ URLs } \\
\hline N & 70 & 83 & 70 & 83 & 70 & 83 \\
Media & 208,36 & 108,87 & 49,96 & 58,34 & 23,60 & 27,05 \\
Desv. Est. & 137,67 & 57,89 & 25,27 & 33,66 & 15,68 & 19,54 \\
Mínimo & 49 & 12 & 8 & 7 & 0 & 1 \\
Máximo & 927 & 280 & 159 & 174 & 60 & 90 \\
P25 & 137 & 61 & 34,75 & 32 & 11,75 & 13 \\
P50 & 173 & 102 & 50,50 & 55 & 20 & 24 \\
P75 & 252,50 & 141 & 66 & 80 & 34,25 & 35 \\
\hline
\end{tabular}

La mayor frecuencia de uso de la asignatura virtualizada en el curso 2019/20 se registró a partir de las 15 horas, coincidiendo con la finalización de las clases y de los talleres prácticos presenciales. Los valores obtenidos indican una actividad mayor en BM que en la media de las asignaturas virtualizadas de la titulación y que en el total de espacios virtualizados en la UCM. Se constata un patrón horario bastante similar al comparar ambos cursos, con un descenso en la actividad registrada en el curso 2019/20 (Fig.1).

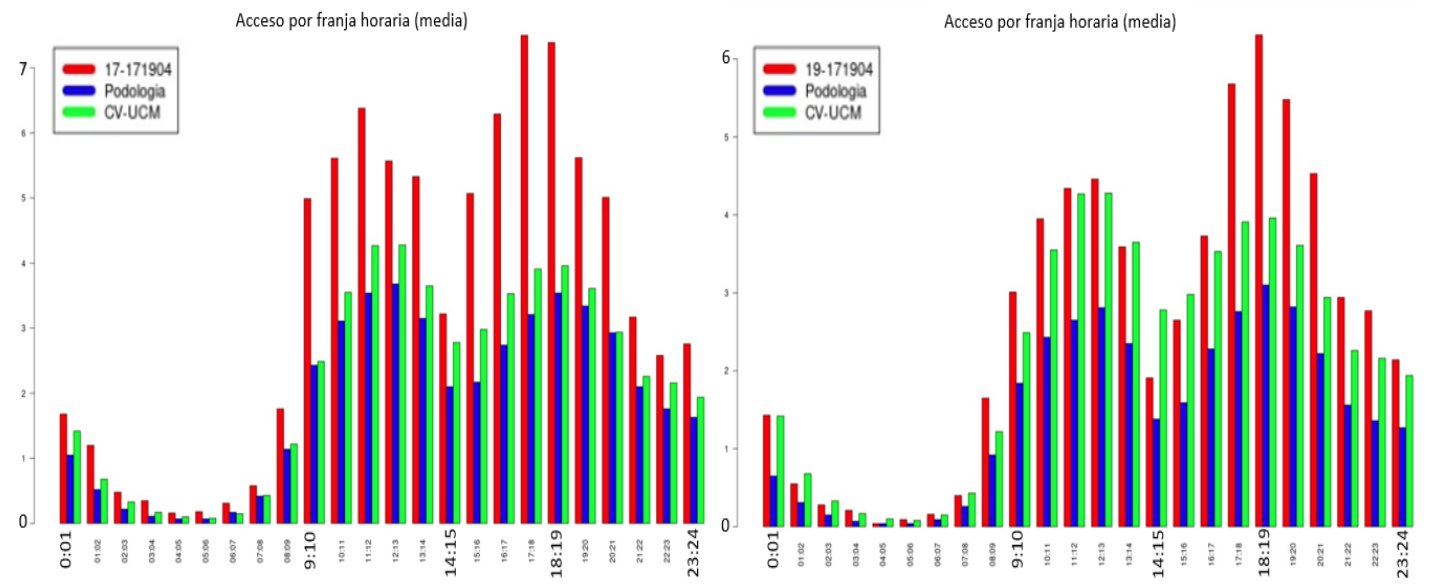

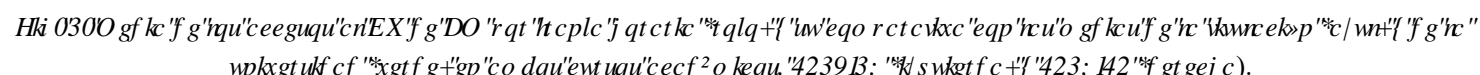

El descenso de la actividad registrada en el CV de BM en el curso 2019/20 se observa asimismo en los mapas de color (Fig.2). Los estudiantes de BM durante el curso 2017/18 presentaron un número de accesos 
muy superior, con pico máximo de 488 visitas registradas en un día, mientras que en el curso 2019/20 no se superó el máximo de las 300 visitas a lo largo del semestre.

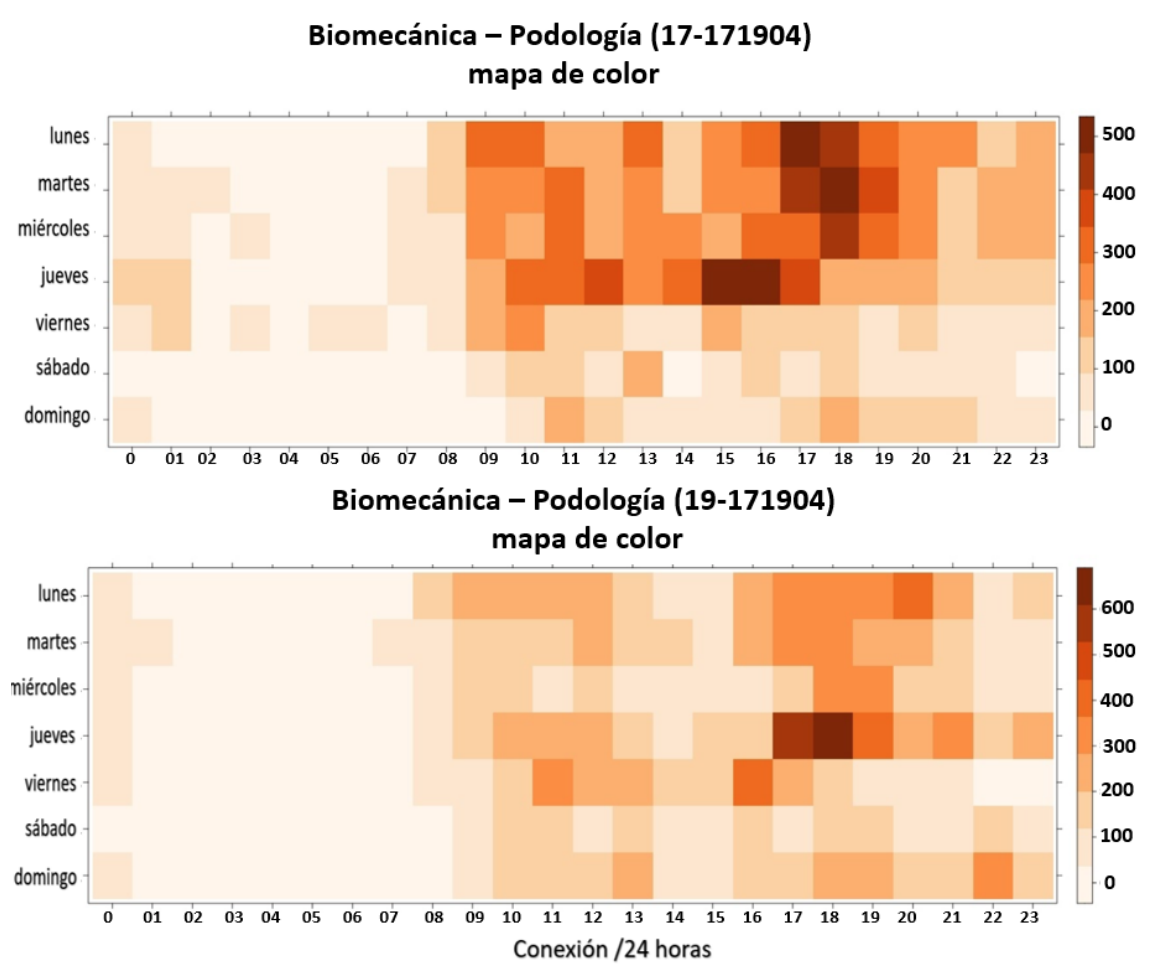

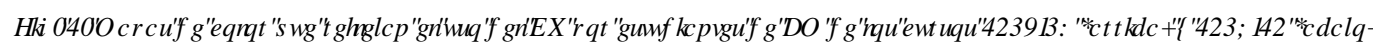

El descenso en los registros de la actividad en el CV contrasta con la mejora en el rendimiento académico en el curso 2019/20, al obtenerse un menor número de suspensos y calificaciones finales más altas. Además, el porcentaje de "no presentados" en la convocatoria de febrero disminuyó hasta el 7,2\% frente al 15,71\% en el curso 2017/18.

Cuando se analiza el comportamiento desglosado por grupos de estudiantes según sus calificaciones finales, las diferencias entre ambos cursos son evidentes (Fig. 3). Así, en el curso 2017/18, todos los grupos de estudiantes fuera cual fuera su categoría por nota mostraron una media de visitas al CV muy elevada, hasta el punto de que la media de los accesos del grupo con nota suspenso fue similar a la realizada en el curso 2019/20 por el grupo de estudiantes con nota de sobresaliente. Sin embargo la media de uso de los recursos en el primer curso fue notoriamente inferior a la efectuada por cualquier grupo de estudiantes en el segundo curso. Nótese que la media del uso de recursos del grupo con mejores notas (entre 8 y 10) en el curso 2019/20 casi duplica la efectuada por el grupo con mejores notas (entre 5 y 8 ) del curso 2017/18. 
Cluster por grupos de Notas - Biomecánica - Podología (17-171904)

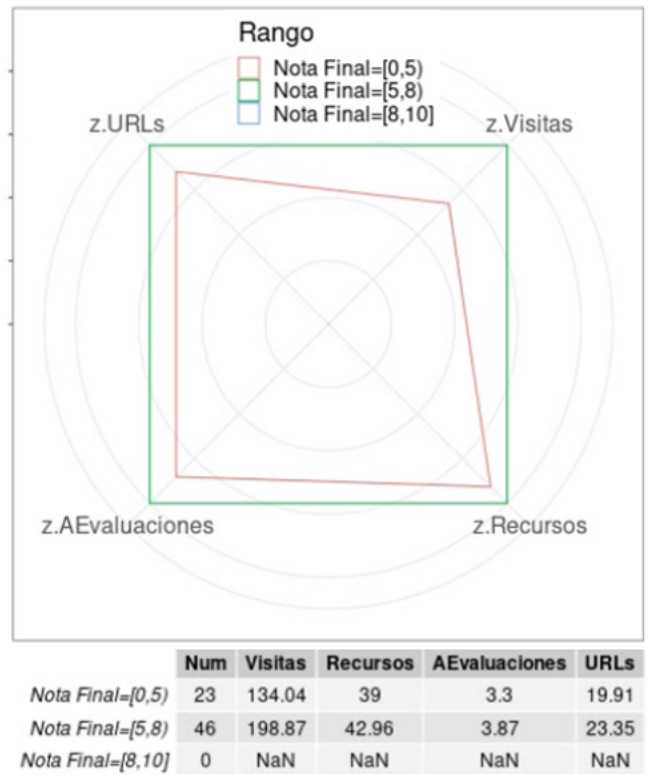

Cluster por grupos de Notas - Biomecánica - Podología $(19-171904)$

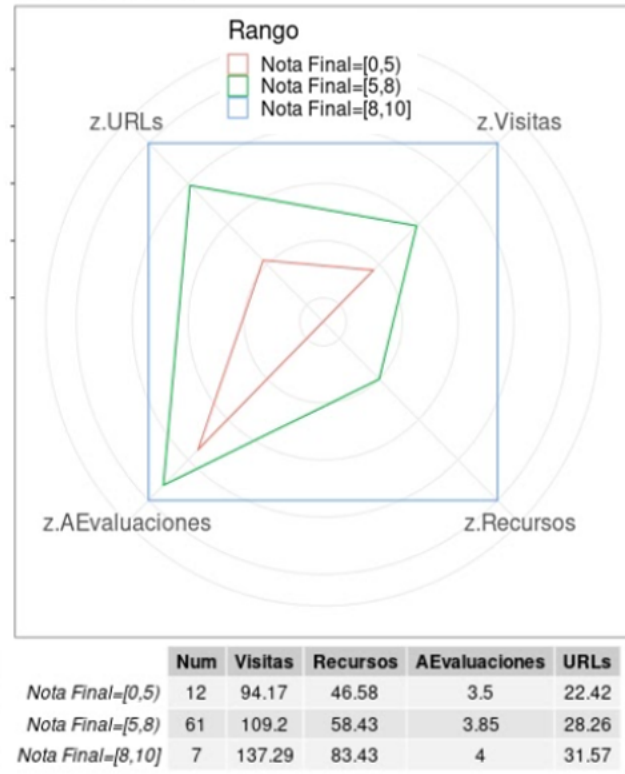

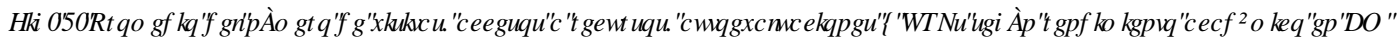

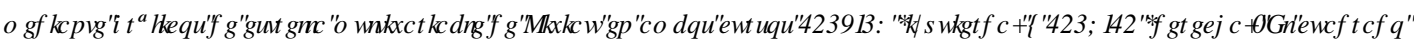 H UHURUUБLHНQMHDP i [IP RIHQFDODFXURIDFDQPP IFRD}

Al buscar relaciones entre el uso del CV y las calificaciones obtenidas, el estadístico de Spearman reveló en el curso 2019/20 que la calificación final de la asignatura, correspondiente a la convocatoria de febrero, y también la calificación de los cuestionarios de evaluación correlacionaban significativamente con el número de cuestionarios de evaluación realizados en el $\mathrm{CV}(\mathrm{r}=0,263 ; \mathrm{p}<0,05 \mathrm{y} \mathrm{r}=0,436 ; \mathrm{p}<0,001$, respectivamente); así mismo, esta última calificación y la nota final de prácticas correlacionaban significativamente con el uso de los recursos de la plataforma virtualizada $(r=0,266$ y $r=0.253 ; p<0,05)$. Sin embargo, en el curso 2017/18 no se observaron correlaciones significativas entre el uso del CV y las calificaciones final, teórica y práctica.

El estudio mediante árboles de decisión indicó claras diferencias entre ambos cursos (Fig. 4). En el curso 2017/18, el árbol obtenido para la calificación final de la asignatura en la convocatoria de febrero siguiendo el método CHAID (calidad estimada 1,539), mostró dos nodos en función de que el número de visitas superara las 145 pero con un valor $\mathrm{p}$ no significativo, lo que indica que la variable no tuvo un efecto estadísticamente significativo en la calificación final de la convocatoria de febrero. En cambio, en el curso 2019/20 el árbol obtenido con el método CHAID (calidad estimada 2,151), mostró dos nodos según el número de cuestionarios completados con un valor de $\mathrm{p}=0,041$ de forma que, los estudiantes del nodo 2 obtuvieron mejores calificaciones en el acta de febrero que los del nodo 1 (nota media superior en 1 punto). La segunda variable que parece influir en las calificaciones finales fue el número de visitas al CV, si bien no fue estadísticamente significativa $(\mathrm{p}=0,13)$. Los nodos 3 y 4 que se obtienen teniendo en cuenta esa segunda variable recuerdan a lo descrito el curso 2017/18: un mayor número visitas se acompañó de peores resultados académicos. Así pues, los individuos del nodo 4, que proceden del grupo que hizo menos de 3 cuestionarios, son los que mostraron una media mayor de accesos al CV y cosecharon peores notas finales, en comparación con lo del nodo 3. 


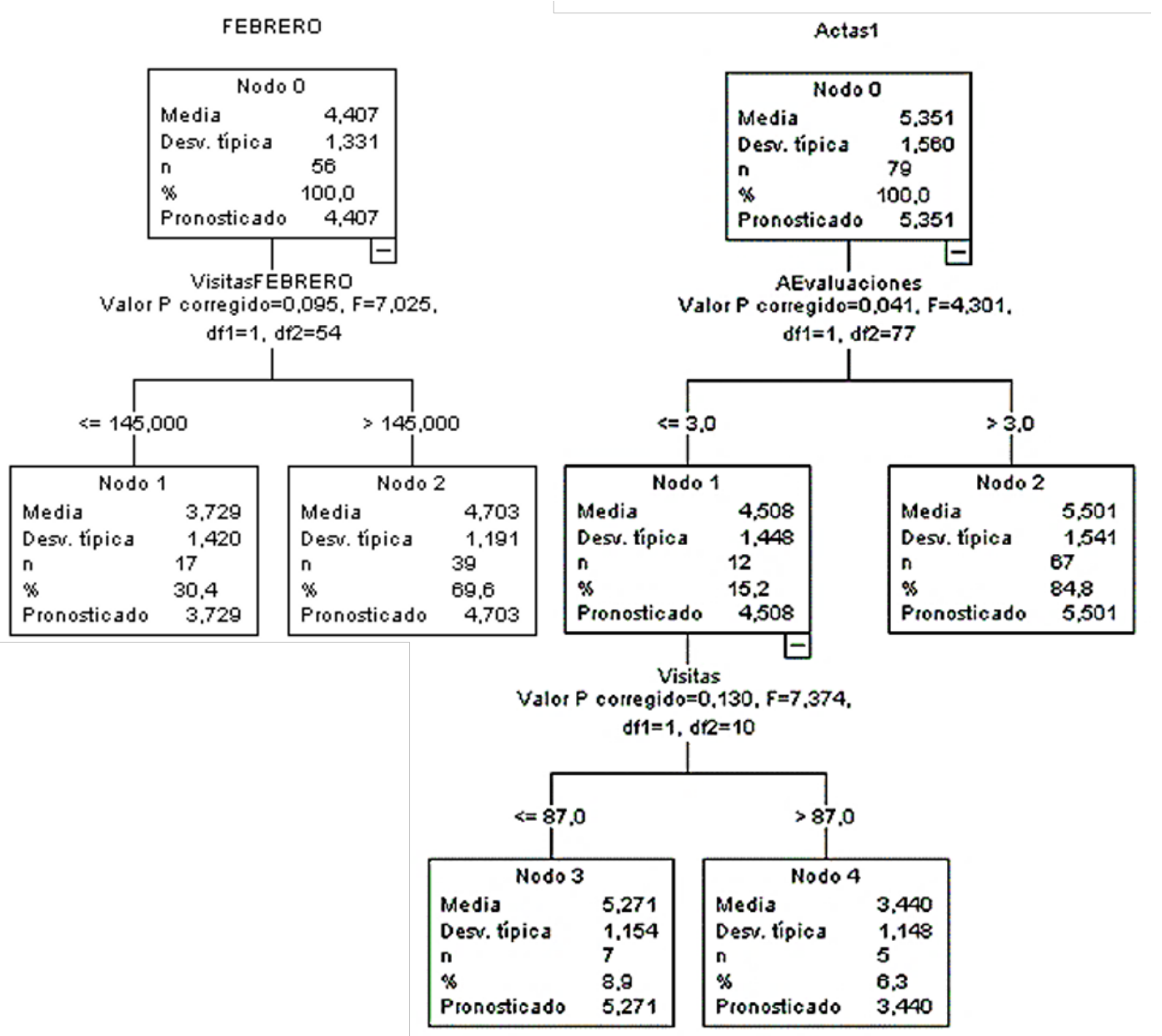

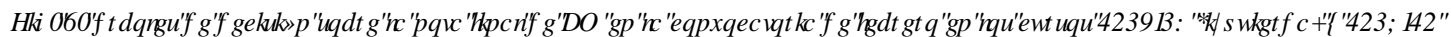 $\square$ प}

Los árboles obtenidos para la nota final de prácticas en la primera convocatoria según el método CHAID se muestran en la Figura 5. En el curso 2017/18 se obtuvieron dos nodos en función de que el número de visitas superara las 141 pero nuevamente, como ocurría con la nota final de la asignatura, la variable visitas no fue estadísticamente significativa en la calificación práctica final del estudiante. Por el contrario, en el curso 2019/20, la variable visitas permite obtener dos nodos con diferencias estadísticamente significativas ( $\mathrm{p}=0,039)$ de forma que, los estudiantes del nodo 2 (número de accesos superior a 98) obtuvieron mejores notas en el examen de prácticas que los del nodo 1 (nota media superior en 1,2 puntos). 


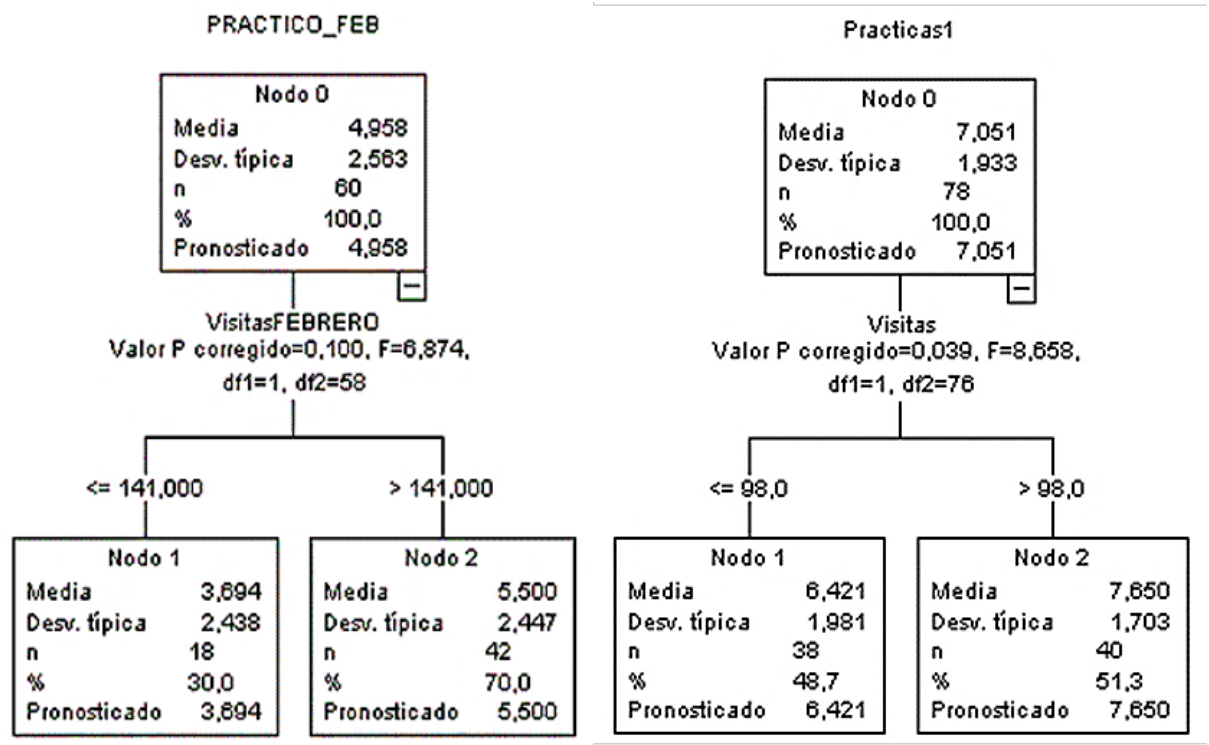

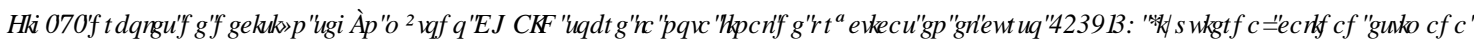

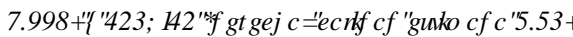

Los estadísticos de las notas obtenidas por el alumnado durante los cursos 2017/18 y 2019/20, se recogen en la Tabla 2. Se agrupan según nota final de febrero ( $\$ F W D$ ), prácticas de febrero (3U FUFDV ), teoría de febrero (7HRED ) y calificación global (Final_T) de la asignatura (convocatorias de febrero y julio).

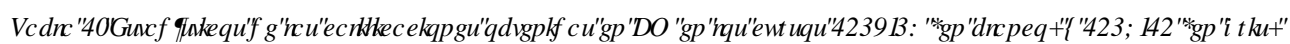

\begin{tabular}{lccc}
\hline & N & Media & $\begin{array}{r}\text { Desviación } \\
\text { estandar }\end{array}$ \\
Final_T & 64 & 5,139 & 1,3464 \\
& 79 & 5,889 & 1,3866 \\
\hline Actas1 & 56 & 4,407 & 1,3307 \\
& 79 & 5,351 & 1,5597 \\
\hline Prácticas1 & 60 & 4,96 & 2,563 \\
& 78 & 7,05 & 1,933 \\
\hline Teoría1 & 56 & 4,040 & 1,3874 \\
& 76 & 5,134 & 1,5009 \\
\hline
\end{tabular}

Al comparar las calificaciones de teoría, prácticas y global de la asigantura de ambos cursos académicos se observa que existen diferencias significativas en los resultados obtenidos por el alumnado (t-Student y Mann-Withney, $\mathrm{p}<0,001$ ), siendo éstas mayores en el curso 2019/2020. 


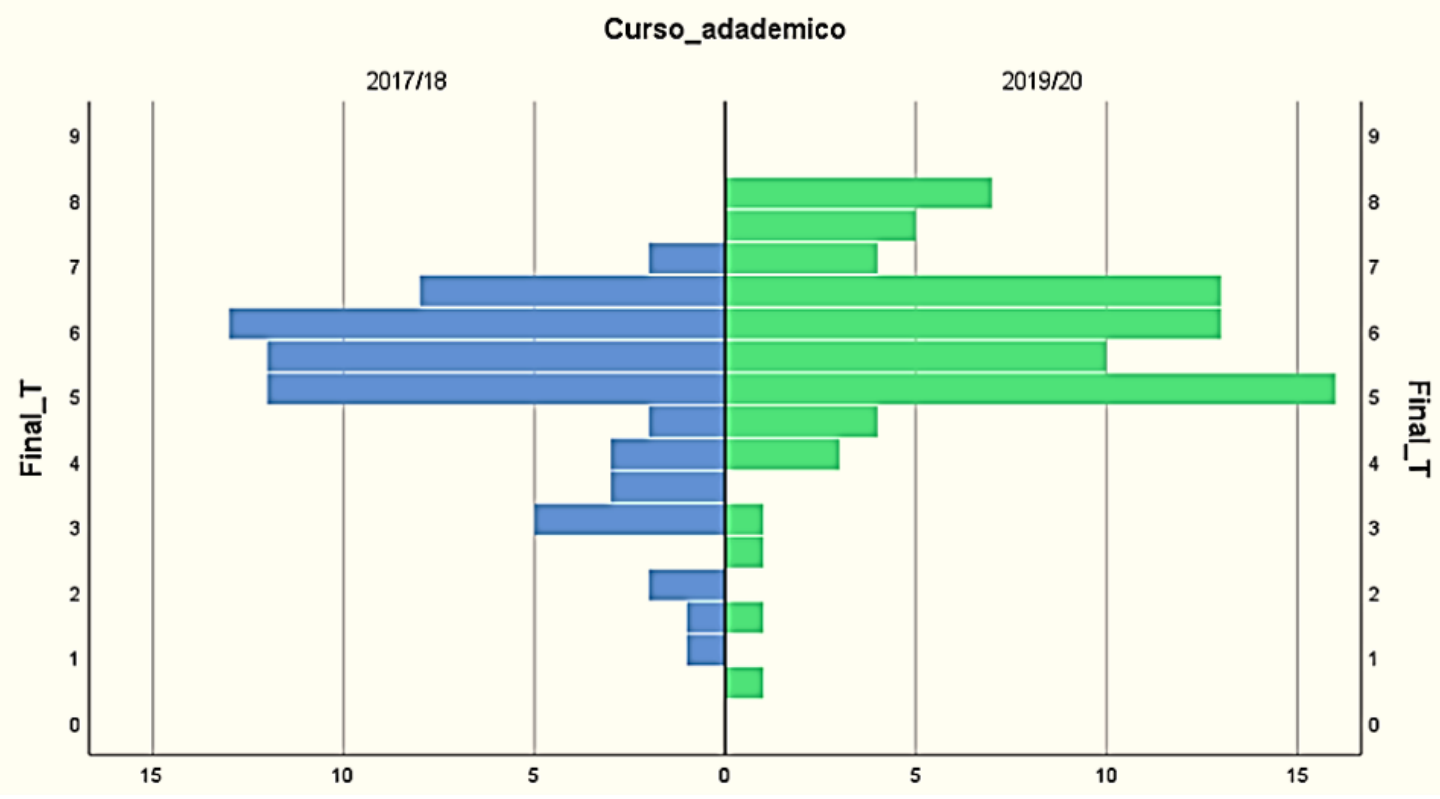

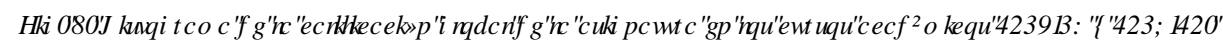

Para completar el estudio, se compararon las calificaciones finales de la asignatura BM, suma de las notas de las convocatoria de febrero y julio de ambos cursos académicos (Fig.6). Se encontraron diferencias estadísticamente significativas entre ambos (t-Student, $p=0,001$; Mann-Withney, $p=0,002$ ), siendo mayores las calificaciones en el curso 2019/2020.

\section{Conclusiones}

En este estudio hemos podido constatar que los cambios introducidos en la docencia teórica y práctica en el curso 2019/20 de la asignatura BM han producido mejores rendimientos académicos, tanto en las notas de teoría y de prácticas como en la calificación final de la asignatura, respecto del curso 2017/18. La incorporación de las autoevaluaciones del contenido teórico ha incidido en un mejor rendimiento de aquellos que las completaron, mientras que la utilización del aula invertida en la materia práctica ha mejorado la nota en el examen final práctico final de la materia, comprobando en ambos casos, que el alumnado del curso 2019/20 ha hecho un uso más racional del CV. Además, la innovación desarrollada no sólo se ha traducido en una mejora en el aprendizaje sino que ha supuesto un estímulo para que un mayor número de estudiantes se presentase a los exámenes, disminuyendo el número de "no presentados".

El estudio realizado del uso del CV y del rendimiento académico de la asignatura BM durante el curso académico 2017/18 había mostrado que un número elevado de visitas al CV no se traducía en mayor uso de los recursos que el profesorado incluía en la asignatura virtualizada ni en un buen rendimiento académico (Angulo et al., 2020). Este comportamiento errático de los estudiantes de BM en el CV durante el curso 2017/18 nos hizo convenir con autores como Mwalumbwe y Mtebe (2017) y Rosetti et al. (2017) en que algunas de las variables de los registros como son el tiempo de uso, el número de accesos a la asignatura virtualizada e de interacción con recursos y actividades, no siempre tienen un impacto significativo en el rendimiento de los estudiantes. Partimos del hecho de que nuestros estudiantes son nativos digitales, pero esto no es óbice para que, a la vez, carezcan de ciertas competencias en el buen uso académico de las TIC. Autores como Popescu et al. (2019) sugieren que los educadores deben interactuar más con la nueva 
generación de estudiantes, estimular debates en las clases, introducir metodologías activas como el aula inversa o incorporar actividades prácticas, hacer la docencia más visual y proporcionar de forma rápida retroalimentación.

Los resultados de Borgobello y Roselli (2016) y Jenaro et al. (2018), que sugieren que para obtener un buen rendimiento académico se precisa la implicación activa del estudiante y no sólo el simple acceso a la información disponible, nos orientaron a implementar la metodología docente para tratar revertir los malos resultados obtenidos por el alumnado de BM en el curso 2017/18. Para ello, nos propusimos trabajar en una doble diección. Por un lado, realizar un refuerzo activo de los contenidos teóricos mediante evaluaciones formativas, esto es, autoevaluaciones realizadas en el CV al terninar cada unidad didáctica para conocer y trabajar in situ los conceptos más complejos y que peor asimilan los estudiantes (Martínez, 2012). Por otro, en el caso del contenido práctico de la materia, nos inclinamos por metodología del aula inversa la cual favore el aprendizaje colaborativo entre iguales, fomenta la compresión más profunda de los conceptos y estimula la construcción de la inteligencia colectiva (Fidalgo-Blanco et al., 2017; Sein-Echaluce et al., 2017; Álvarez et al., 2018; Prieto-Martín et al., 2019). Los resultados del curso 2019/20 han sido claramente satisfactorios en ambos casos.

Es indudable que el conocimiento de las características de las nuevas generaciones que acceden al ámbito universitario, de sus hábitos de estudio y de sus preferencias como grupo, orientan al profesorado a la hora de elegir las herramientas y la metodología docente adecuada en beneficio de mejorar el aprendizaje del estudiante (Mallén y Domínguez, 2014). En nuestro caso, el análisis de los $\mathbb{R} J V$ del CV almacenados en Moodle nos permitió abrir una reflexión sobre el comportamiento de los estudiantes en el CV, su relación con el rendimiento académico y buscar la forma de mejor ambos aspectos. Así pues, conocer la información que guardan los registros de la actividad de los estudiantes puede ayudar a la toma de decisiones fundadas para revertir malos hábitos y mejorar el aprendizaje del alumnado.

\section{Agradecimientos}

Este estudio forma parte del proyecto Innova-Docencia titulado "Estudiantes y Campus Virtual. Utilidad del Learning Analytics para identificar luces y sombras y caminar hacia metodologías adaptativas" concedido por la UCM en el curso académico 2019/20.

\section{Referencias}

Álvarez Sagubay, P.J., Toapaxi Acosta, C.V., Reyes Wagnio, M.F. y Quinzo Bravo, M.C. (2018). "La metodología flip teaching un nuevo entorno de aprendizaje" en 5HILW0 DJDIQHCHCW\&IHQFDV, 3(2), p. 95102. $<$ https://revistas.utb.edu.ec/index.php/magazine/article/view/495/367 $>$ [Consulta: 20 de mayo de 2021]

Angulo Carrere, M.T. M.T., Álvarez-Méndez, A.M., Bravo-Llatas, M.C., Cristóbal Barrios, J. y Álvarez VÁzQUEZ, M.P. (2020). "Diferencias de comportamiento entre estudiantes de dos asignaturas de CC. de la

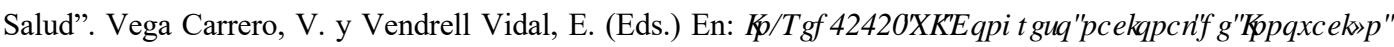

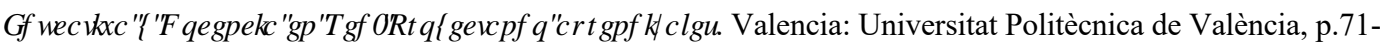
82. $<$ http://dx.doi.org/10.4995/INRED2020.2020.11940 > [Consulta: 22 de marzo de 2021]

Borgobello, A. y Roselli, N.D. (2016). "Rendimiento académico e interacción sociocognitiva de estudiantes en un entorno virtual" en (GXFDomR $\quad H \quad$ 3НTXIMD, $42 \quad$ (2), p. $359-374$. $<\underline{\text { http://www.scielo.br/scielo.php?script=sci arttext\&pid=S1517-97022016000200359 }}>[$ Consulta: 22 de marzo de 2021] 
Chaо ChaO, K.-W. (2014). "Estrategias didácticas mediadas con TIC en un curso de expresión oral francesa" en

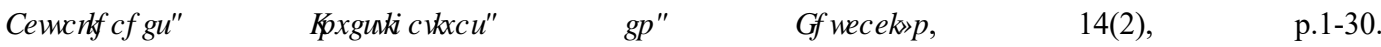
$<$ https://www.scielo.sa.cr/pdf/aie/v14n2/a07v14n2.pdf $>$ [Consulta: 22 de marzo de 2021]

Ferrero, R. y LóPeZ, J.L. (2018). "Data Science. ¿Qué es R software? en el Blog - 0 i [IPD]) RLPDFly 2018. $<$ https://www.maximaformacion.es/blog-dat/que-es-r-software/> [Consulta: 22 de marzo de 2021]

Fidalgo-Blanco, A. Martinez-Nuñez, M. Borrás-Gene, O. SÁnchez Medina, J. J. (2017). "Micro flip teaching An innovative model to promote the active involvement of students" en \&RP SXWLWIQ+XPDQ\% KDYRU 72, p. 713-723. < https://www.sciencedirect.com/science/article/abs/pii/S0747563216305507 > [Consulta: 20 de mayo de 2021]

GARcía AyALA, E. DE J. (2017). "Millennials la nueva generación de profesionalistas del siglo XXI” en 5HLWD\&IHQFID \$ F IQUWDUVD, 1, p.174-183. < https://www.uv.mx/iiesca/files/2017/10/20CA201701.pdf > [Consulta: 22 de marzo de 2021]

GRANAdOS-ZÚÑIGA, J. (2019). "Relación entre el uso del aula virtual y el rendimiento académico en estudiantes del curso de Bioquímica para Enfermería de la Universidad de Costa Rica" en 5HLWD( GXFDFlyQ 43(2), p.1-16. < https://www.scielo.sa.cr/pdf/edu/v43n2/2215-2644-edu-43-02-00327.pdf > [Consulta: 22 de marzo de 2021]

Huapaya, C.R. Lizarralde, F.A., Arona, G.M. y Massa, S.M. (2012). "Minería de Datos Educacional en Ambientes Virtuales de Aprendizaje" en ; ,9!: RLMKRSLCH, QYHWDDRLHKHQ\&HQFLVGHCD\&RP SXWFIYQ 1, p. 996-1000.

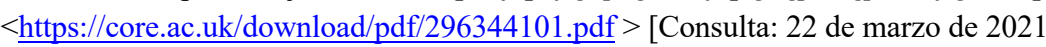

Jenaro Río, C., Castaño Calle, R., Martín Pastor, M.E., y Flores Robaina. N. (2018). "Rendimiento académico en educación superior y su asociación con la participación activa en la plataforma Moodle" en ( WKGRVUREUH ( GXFDFlyQ 34, p.177-198. < https://revistas.unav.edu/index.php/estudios-sobre-educacion/article/view/8365 > [Consulta: 22 de marzo de 2021]

Mallén Broch, F.F. y Domínguez EsCrig, E. (2014). “Acciones para la mejora del rendimiento académico a través

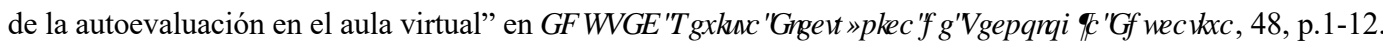
$<$ https://www.edutec.es/revista/index.php/edutec-e/article/view/49 > [Consulta: 22 de marzo de 2021]

MARTínez Rizo, F. (2012). "La evaluación formativa del aprendizaje en el aula en la bibliografía en inglés y francés. Revisión de literatura" en 5HLWD 0 HIFDQD GH, QYHWDFFYQ (GXFDMD, 17(54), p.849-875. < https://www.redalyc.org/articulo.oa?idp=1\&id=14023127008\&cid=7493 $>$ [Consulta: 22 de marzo de 2021]

Mwalumbwe, I. y MteBe, J.S. (2017). “Using Learning Analytics to predict students' performance in Moodle Learning Management System: A case of Mbeya University of Science and Technology" en (-,6' \& 7 KH( OFFRQRE

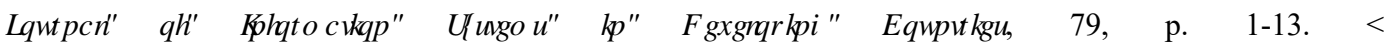
https://onlinelibrary.wiley.com/doi/epdf/10.1002/j.1681-4835.2017.tb00577.x $>$ [Consulta: 22 de marzo de 2021]

OllÉ SÁNCHEZ, J. (2019). “¿Cómo seleccionar y utilizar el mejor software para poder adaptarte a la era de los datos y ser único en tu sector? en el Blog - \&RQFWLRV\&(DURV, 2019. < https://conceptosclaros.com/software-analisis-

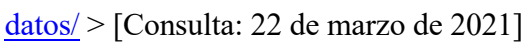

Pernías, P. (2011). "Los Campus Virtuales como favorecedores del aprendizaje de los estudiantes. El caso de la

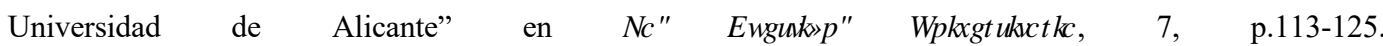
$<$ http://polired.upm.es/index.php/lacuestionuniversitaria/article/view/3391 > [Consulta: 22 de marzo de 2021]

(cc) EY-NC-ND 2021, Universitat Politècnica de València

CRQJHMR, Q5HGTIOTI 
Popescu, D., Popa, D.M. y Cotet, B.G. (2019). "Preparando a los estudiantes para la Generación Z: consideraciones sobre el currículo de impresión 3D” en 3URSy ULRV II 5HLUMHQWFIRQH, 7(2), p.240-268. < http://www.scielo.org.pe/pdf/pyr/v7n2/a10v7n2.pdf $>$ [Consulta: 22 de marzo de 2021]

Prieto-Martín, a. Barbarroja-Escudero, J. Lara-Aguilera, i. Díaz-Martín, D. Pérez-Gómez, A. MontserratSanz, J. Corell-Almuzara, A.Álvarez de Mon-Soto, M. (2019). "Aula invertida en enseñanzas universitarias:

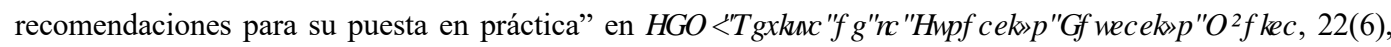
p.253-262. < https://scielo.isciii.es/pdf/fem/v22n6/2014-9832-fem-22-6-253.pdf > [Consulta: 20 de mayo de 2021]

Quirós Meneses, E. (2009). "Recursos didácticos digitales: medios innovadores para el trabajo colaborativo en línea" en 5HLWDHOFUy QFD( GXFDH 13(2), p.47-62 < https://dialnet.unirioja.es/servlet/articulo?codigo=4781052 > [Consulta: 22 de marzo de 2021]

Rosetti López, S.R., Verdugo TAPiA, M.L. y BAylissS Bernal, D. (2017). "Learning Analytics para determinar la relación entre uso de un Learning Management System y rendimiento académico", en ; ; , $\square \& R Q J H R]$

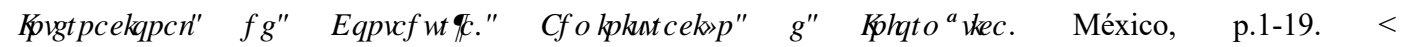

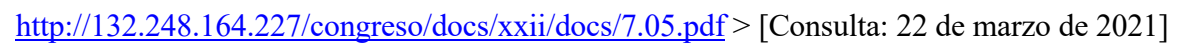

SAEl, N., MARZAK, A. y BEHJA, H. (2013). "Web usage mining data preprocessing and multilevel analysis on Moodle" en ,-\&6,0 ,QMLQDMRQDO -RXLQDO RI \&RPSXWU 6FHQH ,WXH, $10(2), \quad$ p.347-354. <

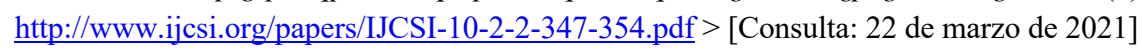

SALINAS IBÁÑEZ, J. (2004). "Entornos virtuales y formación flexible” en 7HFQRQRJLDHQ0 DHFD, 17 (3 Especial), p.6980. $<$ https://dialnet.unirioja.es/servlet/articulo?codigo $=4835450>$ [Consulta: 22 de marzo de 2021]

Sein-Echaluce M.L., Fidalgo-Blanco A. y García-Peñalvo, F.J. (2017). “Trabajo en equipo y Flip Teaching para

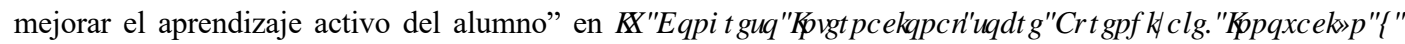
\&RP SHMWMIGDFT\&, 1 \$, \&. Zaragoza. p. 610-615. < https://zaguan.unizar.es/record/62959? In=es $>$ [Consulta: 20 de mayo de 2021]

Vinueza Morales, S.X. y Morocho Macas, Á.A. (2017). “Análisis del rendimiento académico en la Cátedra de

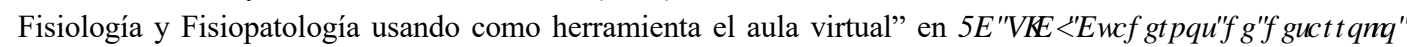
DSQFDORVDDQV7, \&, 6(3), p.43-60. < https://dialnet.unirioja.es/servlet/articulo?codigo=6253715 $>$ [Consulta: 22 de marzo de 2021] 